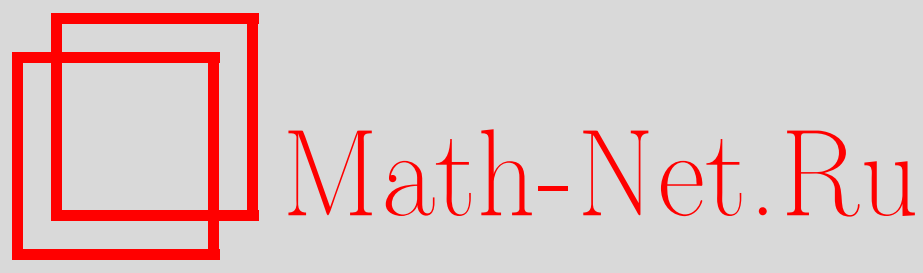

М. Э. Муминов, Н. М. Алиев, О спектре трехчастичного оператора Шредингера на одномерной решетке, ТМФ, 2012, том 171, номер 3, 387-403

DOI: https://doi.org/10.4213/tmf6917

Использование Общероссийского математического портала Math-Net.Ru подразумевает, что вы прочитали и согласны с пользовательским соглашением http://www.mathnet.ru/rus/agreement

Параметры загрузки:

IP : 35.174 .16 .151

26 апреля 2023 г., 13:34:08

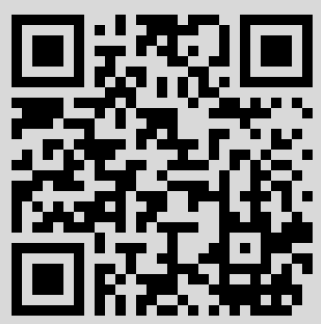




\title{
О СПЕКТРЕ ТРЕХЧАСТИЧНОГО ОПЕРАТОРА ШРЕДИНГЕРА НА ОДНОМЕРНОЙ РЕШЕТКЕ
}

\begin{abstract}
Рассматривается система трех произвольных квантовых частиц на одномерной решетке, взаимодействующих с помощью парных контактных потенциалов притяжения. Доказывается конечность дискретного спектра соответствующего оператора Шредингера при всех значениях полного квазиимпульса в случае, когда массы двух частиц конечны. Показывается бесконечность дискретного спектра оператора Шредингера в случае, когда в трехчастичной системе массы двух частиц бесконечны.
\end{abstract}

Ключевые слова: трехчастичная система на решетке, оператор Шредингера, существенный спектр, дискретный спектр, компактный оператор.

\section{1. ВВЕДЕНИЕ}

Существование бесконечного числа собственных значений, накапливающихся к левому краю существенного спектра оператора Шредингера (эффект Ефимова [1]), изучалось во многих физических работах [2], [3]. Строгое математическое доказательство существования эффекта Ефимова впервые было проведено в работе Яфаева [4] с использованием методов интегральных уравнений Фаддеева. Затем существование бесконечного числа связанных состояний было установлено в работах [5], [6] и для гамильтониана системы трех частиц, взаимодействующих с помощью парных контактных потенциалов притяжения, доказано в работах [7], [8] при нулевом значении полного квазиимпульса системы. Конечность связанных состояний дискретного оператора Шредингера трех частиц доказывалась в работах [8], [9] при малых значениях полного квазиимпульса $K \neq 0$. В работе [10] найдено условие появления лакуны существенного спектра и доказано существование бесконечного числа собственных значений в этой лакуне для дискретного оператора Шредингера трех частиц.

Заметим, что в работах [7], [8] бесконечное число собственных значений появляется ниже существенного спектра в случае, когда два из трех двухчастичных подгамильтонианов имеют виртуальные уровни в нуле и нуль является регулярной

* Самаркандский государственный университет, Самарканд, Узбекистан.

E-mail: mmuminov@mail.ru 
точкой для третьего гамильтониана или все двухчастичные подгамильтонианы имеют виртуальные уровни в нуле.

В настоящей работе рассматривается система трех произвольных частиц на одномерной решетке, взаимодействующих с помощью парных контактных потенциалов притяжения. Изучаются спектральные свойства соответствующего оператора Шредингера $H(K), K \in T=(-\pi, \pi]$. Доказывается конечность дискретного спектра, лежащего левее существенного спектра соответствующего оператора Шредингера, при всех значениях энергии взаимодействия $\mu_{\alpha}>0$ двух частиц $\beta$ и $\gamma$ системы трех частиц и при всех значениях полного квазиимпульса $K \in T$ в случае, когда массы хотя бы двух частиц конечны.

Отметим, что существенный спектр $\sigma_{\text {ess }}(H(K))$ оператора $H(K)$ состоит из объединения спектров канальных операторов $H_{\alpha}(K), \alpha=1,2,3$. В отличие от трехчастичного оператора Шредингера на трехмерной решетке спектр канального оператора $H_{\alpha}(K)$ на одномерной решетке при всех значениях энергии взаимодействия $\mu_{\alpha}>0$ (а не определенных значениях $\mu_{\alpha}>0$ ) состоит из объединения спектра свободного гамильтониана $H_{0}(K)$ и некоторого отрезка $\left[\lambda_{\alpha}^{\min }(K), \lambda_{\alpha}^{\max }(K)\right]$, т. е.

$$
\sigma\left(H_{\alpha}(K)\right)=\sigma\left(H_{0}(K)\right) \cup\left[\lambda_{\alpha}^{\min }(K), \lambda_{\alpha}^{\max }(K)\right] .
$$

С увеличением $\mu_{\alpha}>0$ этот отрезок сдвигается влево от $m_{K}$, а с уменьшением $\mu_{\alpha}>0$ приближается к $m_{K}$, где $m_{K}$ - левый край спектра свободного гамильтониана.

Доказано, что при $m_{\alpha}=\infty, m_{\beta}=\infty$ и $m_{\gamma}<\infty$ отрезок $\left[\lambda_{i}^{\min }(K), \lambda_{i}^{\max }(K)\right]$, $i=\alpha, \beta$, превращается в точку, т. е. $\lambda_{i}=\lambda_{i}^{\min }(K)=\lambda_{i}^{\max }(K), i=\alpha, \beta$. В этом случае число $\lambda_{i}$ является бесконечнократным собственным значением канального оператора $H_{i}(K), i=\alpha, \beta$, и появляется бесконечное число собственных значений оператора $H(K)$, стремящихся к $\lambda_{i}, i=\alpha, \beta$. Вычисляются эти собственные значения и их кратности. Показано, что существование таких собственных значений не зависит от $\mu_{\gamma}>0$. Поскольку структура существенного спектра имеет вид

$\sigma_{\text {ess }}(H(K))=\sigma\left(H_{0}(K)\right) \cup\left\{\lambda_{\alpha}\right\} \cup\left\{\lambda_{\beta}\right\} \cup\left[\lambda_{\gamma}^{\min }(K), \lambda_{\gamma}^{\max }(K)\right], \quad \lambda_{\gamma}^{\min }(K)<\lambda_{\gamma}^{\max }(K)$,

наблюдается интересная картина: при некоторых значениях $\mu_{\gamma}>0$ бесконечное число собственных значений оператора $H(K)$ появляется в существенном спектре, точнее, в отрезке $\left[\lambda_{\gamma}^{\min }(K), \lambda_{\gamma}^{\max }(K)\right]$, а при больших значениях $\mu_{\gamma}>0$ найденные собственные значения могут находиться на лакуне (на интервале $\left(\lambda_{\gamma}^{\max }(K), m_{K}\right)$ ) существенного спектра оператора Шредингера.

\section{2. ОПИСАНИЕ ТРЕХЧАСТИЧНОГО ОПЕРАТОРА И ФОРМУЛИРОВКА ОСНОВНЫХ РЕЗУЛЬТАТОВ}

Пусть $Z$ - одномерная целочисленная решетка, т. е. множество целых чисел, и $\ell_{2}\left(Z^{3}\right)$ - гильбертово пространство квадратично-суммируемых функций, определенных на $Z^{3}$.

Свободный гамильтониан $\widehat{H}_{0}$ системы трех произвольных квантовых частиц с дисперсионными соотношениями $\hat{\varepsilon}_{i}, i=1,2,3$, на решетке $Z$ определяется как ограниченный самосопряженный оператор в гильбертовом пространстве $\ell_{2}\left(Z^{3}\right)$ :

$$
\begin{aligned}
\left(\widehat{H}_{0} \hat{\psi}\right)\left(n_{1}, n_{2}, n_{3}\right)= & \sum_{s \in Z}\left[\hat{\varepsilon}_{1}(s) \hat{\psi}\left(n_{1}+s, n_{2}, n_{3}\right)+\hat{\varepsilon}_{2}(s) \hat{\psi}\left(n_{1}, n_{2}+s, n_{3}\right)+\right. \\
& \left.+\hat{\varepsilon}_{3}(s) \hat{\psi}\left(n_{1}, n_{2}, n_{3}+s\right)\right]
\end{aligned}
$$


где $\hat{\varepsilon}_{i}(s)=\hat{\varepsilon}(s) / 2 m_{\alpha}, \alpha=1,2,3, m_{\alpha}>0$ - масса частицы $\alpha, \hat{\varepsilon}$ - элемент пространства $\ell_{1}(Z)$, описывающий перенос частицы с узла на соседний узел, определенный в $Z$ по формуле

$$
\hat{\varepsilon}(s)= \begin{cases}\frac{2}{\sqrt{2 \pi}}, & s=0, \\ \frac{1}{\sqrt{2 \pi}}, & |s|=1, \\ 0, & |s|>1 .\end{cases}
$$

Трехчастичный гамильтониан $\widehat{H}$ системы трех квантовых частиц с парными контактными потенциалами определяется как ограниченное возмущение свободного гамильтониана $\widehat{H}_{0}$ :

$$
\widehat{H}=\widehat{H}_{0}-\mu_{1} \widehat{V}_{1}-\mu_{2} \widehat{V}_{2}-\mu_{3} \widehat{V}_{3},
$$

где $\left(\widehat{V}_{\alpha} \psi\right)\left(n_{1}, n_{2}, n_{3}\right)=\delta_{n_{\beta} n_{\gamma}} \psi\left(n_{1}, n_{2}, n_{3}\right),\{\alpha, \beta, \gamma\}=\{1,2,3\}$. Здесь $\mu_{\alpha}>0$ - энергия взаимодействия двух частиц, $\delta_{n m}-$ символ Кронекера.

Пусть $L_{2}\left(T^{n}\right), n=1,2,3$, - гильбертово пространство квадратично-интегрируемых функций, определенных на $T^{n}, T=(-\pi, \pi]$.

Используя преобразование Фурье и разложение в прямой операторный интеграл [8], [11], изучение спектральных свойств оператора $\widehat{H}$ можно свести к исследованию спектральных свойств семейства самосопряженных ограниченных операторов $H(K), K \in T$, действующих в гильбертовом пространстве $L_{2}\left(T^{2}\right)$ по формуле

$$
H(K)=H_{0}(K)-V, \quad V=\mu_{1} V_{1}+\mu_{2} V_{2}+\mu_{3} V_{3},
$$

где $H_{0}(K)$ - оператор умножения на функцию

$$
\mathcal{E}_{K}(x, y)=\frac{1}{m_{1}} \varepsilon(x)+\frac{1}{m_{2}} \varepsilon(y)+\frac{1}{m_{3}} \varepsilon(K-x-y), \quad \varepsilon(x)=1-\cos x,
$$

И

$$
\begin{gathered}
\left(V_{1} f\right)(x, y)=\frac{1}{2 \pi} \int_{-\pi}^{\pi} f(x, s) d s, \quad\left(V_{2} f\right)(x, y)=\frac{1}{2 \pi} \int_{-\pi}^{\pi} f(s, y) d s \\
\left(V_{3} f\right)(x, y)=\frac{1}{2 \pi} \int_{-\pi}^{\pi} f(s, x+y-s) d s, \quad f \in L_{2}\left(T^{2}\right)
\end{gathered}
$$

Лемма 1. Для любого $K \in T^{3}$ существенный спектр $\sigma_{\text {ess }}(H(K))$ оператора $H(K)$ состоит из обгединения спектров операторов каналов $H_{\alpha}(K)=H_{0}(K)-$ $\mu_{\alpha} V_{\alpha}, \alpha=1,2,3$, m.e.

$$
\sigma_{\text {ess }}(H(K))=\sigma\left(H_{1}(K)\right) \cup \sigma\left(H_{2}(K)\right) \cup \sigma\left(H_{3}(K)\right) .
$$

Доказательство леммы можно найти в работе [11].

Пусть

$$
m_{K}=\min _{x, y} \varepsilon_{K}(x, y), \quad M_{K}=\max _{x, y} \varepsilon_{K}(x, y) .
$$


Определим при каждом $z \notin\left[m_{K}, M_{K}\right]$ следующие функции:

$$
\begin{aligned}
& \Delta_{1}(x ; z)=1-\frac{\mu_{1}}{2 \pi} \int_{-\pi}^{\pi} \frac{d s}{\overline{\mathcal{E}_{K}(x, s)-z},} \\
& \Delta_{2}(x ; z)=1-\frac{\mu_{2}}{2 \pi} \int_{-\pi}^{\pi} \frac{d s}{\mathcal{E}_{K}(s, x)-z}, \\
& \Delta_{3}(x ; z)=1-\frac{\mu_{3}}{2 \pi} \int_{-\pi}^{\pi} \frac{d s}{\overline{\mathcal{E}_{K}(s, x-s)-z} .}
\end{aligned}
$$

Положим

$$
\begin{gathered}
\sigma_{\alpha}(K)=\left\{z: \Delta_{\alpha}(x ; z)=0 \quad \text { хотя бы для одного } \quad x \in T\right\}, \\
\lambda_{\alpha}^{\min }(K)=\inf \sigma_{\alpha}(K), \quad \lambda_{\alpha}^{\max }(K)=\sup \sigma_{\alpha}(K) .
\end{gathered}
$$

Имеет место равенство [10], [11]

$$
\sigma\left(H_{\alpha}(K)\right)=\left[m_{K}, M_{K}\right] \cup \bigcup_{p \in T^{3}} \sigma_{\text {disc }}\left(h_{\alpha}(K-p)\right),
$$

где $h_{\alpha}(k), k \in T,-$ оператор, действующий в $L_{2}(T)$ по формуле

$$
h_{\alpha}(k)=h_{0}^{\alpha}(k)-\mu_{\alpha} v, \quad \alpha \in\{1,2,3\},
$$

$h_{0}^{\alpha}(k)$ - оператор умножения на функцию $\varepsilon(p) / m_{\beta}+\varepsilon(k-p) / m_{\gamma}$ и

$$
(v f)(p)=\frac{1}{2 \pi} \int_{-\pi}^{\pi} f(s) d s .
$$

Элементарное вычисление показывает, что для всех $\mu_{\alpha}>0$ и $k \in T$ оператор $h_{\alpha}(k)$ имеет единственное отрицательное собственное значение. Поэтому справедлива следующая лемма.

Лемма 2. Для любого $\mu_{\alpha}>0 u K \in T$ спектр $\sigma_{\text {ess }}\left(H_{\alpha}(K)\right)$ onepamopa $H_{\alpha}(K)$ состоит из множества

$$
\sigma_{\text {ess }}\left(H_{\alpha}(K)\right)=\left[\lambda_{\alpha}^{\min }(K), \lambda_{\alpha}^{\max }(K)\right] \cup\left[m_{K}, M_{K}\right] .
$$

Теперь сформулируем основные результаты настоящей работы.

Теорема 1. Предположим, что $m_{\alpha}<\infty, m_{\beta}<\infty, m_{\gamma} \leqslant \infty,\{\alpha, \beta, \gamma\}=\{1,2,3\}$. Тогда для любого $\mu_{i}, i=1,2,3, u K \in(-\pi, \pi]$ число собственных значений оператора $H(K)$, лежащих левее существенного спектра, конечно.

В дальнейшем мы предполагаем, что $m_{1}=\infty, m_{2}=\infty$ и $m_{3}<\infty$. При этом

$$
\mathcal{E}_{K}(p, q)=\frac{1}{m_{3}} \varepsilon(K-p-q) .
$$


Лемма 3. Пусть $\mu_{\alpha}>0, \alpha=1,2, u \mu_{3} \geqslant 0$. Тогда для любого $K \in T$ имеют место равенства

$$
\begin{gathered}
\sigma\left(H_{\alpha}(K)\right)=\left\{\lambda_{\alpha}\right\} \cup\left[0, \frac{2}{m_{3}}\right], \quad \alpha=1,2, \\
\sigma\left(H_{3}(K)\right)=\Lambda_{3} \cup\left[0, \frac{2}{m_{3}}\right]
\end{gathered}
$$

где $\lambda_{\alpha}$ - отрицательное бесконечнократное собственное значениие оператора $H_{\alpha}(K), \alpha=1,2 u \Lambda_{3}=\left\{z: z=\varepsilon(p) / m_{3}-\mu_{3}, \quad p \in T\right\}$. При этом

$$
\begin{array}{ll}
\sigma\left(H_{3}(K)\right)=\left[-\mu_{3}, \frac{2}{m_{3}}\right], & \mu_{3} \leqslant \frac{2}{m_{3}}, \\
\sigma\left(H_{3}(K)\right)=\left[-\mu_{3}, \frac{2}{m_{3}}-\mu_{3}\right] \cup\left[0, \frac{2}{m_{3}}\right], & \mu_{3}>\frac{2}{m_{3}} .
\end{array}
$$

Теорема 2. Пусть $\lambda_{\min }=\inf \left\{\lambda_{1}, \lambda_{2}\right\}, \lambda_{\max }=\sup \left\{\lambda_{1}, \lambda_{2}\right\}$. Для всякого фиксированного $\mu_{1}, \mu_{2}$ и $\mu_{3}$ оператор $H(K)$ имеет бесконечное число собственных значений $\xi_{0}, \xi_{1}, \xi_{2}, \ldots \xi_{n}, \ldots u \zeta_{0}, \zeta_{1}, \zeta_{2}, \ldots \zeta_{n}, \ldots$, лежащих соответственно в $\left(-\infty, \lambda_{\min }\right)$ $u\left(\lambda_{\max }, 0\right)$ maких, что $\lim _{n \rightarrow \infty} \xi_{n}=\lambda_{\min } u \lim _{n \rightarrow \infty} \zeta_{n}=\lambda_{\max }$. Кратность каждого собственного значения $\xi_{n}, n>0\left(\zeta_{n}, n>0\right)$, равна 2 , а $\xi_{0}$ есть простое собственное значение оператора $H(K)$. Точнее,

$$
\lambda_{\alpha}=\frac{1-\sqrt{1+\mu_{\alpha} m_{\alpha}}}{m_{\alpha}}, \quad \alpha=1,2,
$$

а собственное значение $\xi_{n}$ является решением рационального уравнения

$$
\frac{\mu_{1}}{\Delta_{2}(z)} \frac{\mu_{2}}{\Delta_{1}(z)} \frac{m_{3}}{-z} \frac{\left[1-m_{3} z-\sqrt{\left(m_{3} z\right)^{2}-2 m_{3} z}\right]^{2 k}}{-m_{3} z+2}=1, \quad z<\lambda_{\min },
$$

¿əe

$$
\Delta_{\alpha}(z)=1-\frac{\mu_{\alpha} \sqrt{m_{3}}}{\sqrt{2-m_{3} z}} \frac{1}{\sqrt{-z}}, \quad \alpha=1,2 .
$$

Для доказательства теоремы 2 сначала строится инвариантное подпространство $\mathcal{H}_{0}$ относительно оператора $H(K)$, затем изучается спектр оператора $H(K)$ в этом инвариантном подпространстве $\mathcal{H}_{0}$ и его дополнении $L_{2}\left(T^{2}\right) \ominus \mathcal{H}_{0}$. С помощью методов интегральных уравнений показывается существование бесконечного числа собственных значений и определяются соответствующие собственные функции.

ЗАмечание 1 . В теореме 2 числа $\lambda_{\min }$ и $\lambda_{\max }$ не зависят от $\mu_{3}$. Лемма 3 показывает возможность существования таких случаев, когда числа $\lambda_{\min }$ и $\lambda_{\max }$ могут находиться в существенном спектре $H(K)$ или же на лакуне существенного спектра. Например, в случае $\mu_{1}=\mu_{2}$ имеет место $\lambda_{\min }=\lambda_{\max }$. При малых значениях $\mu_{1}$ и $\mu_{3}=2 / m_{3}$ это число находится в подмножестве $\left[-\mu_{3}, 2 / m_{3}\right]$ существенного спектра $\sigma_{\text {ess }} H(K)$, а при больших $\mu_{3}$ - на интервале (на лакуне существенного спектра) $\left(2 / m_{3}-\mu_{3}, 0\right)$. При этом по теореме 2 существуют такие случаи, что оператор $H(K)$ имеет бесконечное число собственных значений в существенном спектре или на лакуне существенного спектра. 


\section{3. КОНЕЧНОСТЬ ДИСКРЕТНОГО СПЕКТРА}

В этом разделе мы предполагаем, что $m_{\alpha}<\infty, m_{\beta}<\infty, m_{\gamma} \leqslant \infty,\{\alpha, \beta, \gamma\}=$ $\{1,2,3\}$.

Пусть

$$
\tau(K)=\inf \sigma_{\text {ess }}(H(K))
$$

и $W_{\alpha}(z)$ - оператор умножения на функцию $\left[\Delta_{\alpha}(x ; z)\right]^{-1}$. Функция $\Delta_{\alpha}(x ; z)$ является положительной при всех $z<\tau(K)$. Поэтому оператор $W_{\alpha}(z)$ положителен при всех $z \in(-\infty, \tau(K))$.

Определим самосопряженный компактный оператор $\mathbf{T}(z), z<\tau(K)$, в гильбертовом пространстве $L_{2}^{(3)}(T)$, заданный матрицей

$$
\mathbf{T}(z)=\left(T_{i j}(z)\right)_{i, j=1}^{3}, \quad T_{i j}(z)= \begin{cases}W_{i}^{1 / 2}(z) K_{i j}(z) W_{j}^{1 / 2}(z), & i \neq j \\ 0, & i=j\end{cases}
$$

Здесь $T_{i j}(z)=W_{i}^{1 / 2}(z) K_{i j}(z) W_{j}^{1 / 2}(z), K_{i j}(z)$ - интегральный оператор, порожденный ядром $K_{i j}(z ; x, s)$ такой, что $K_{i j}(z)=\left[K_{j i}(z)\right]^{*}$, где

$$
\begin{gathered}
K_{12}(z ; x, s)=\frac{(2 \pi)^{-1} \sqrt{\mu_{1} \mu_{2}}}{\mathcal{E}_{K}(x, s)-z}, \quad K_{13}(z ; x, s)=\frac{(2 \pi)^{-1} \sqrt{\mu_{1} \mu_{3}}}{\mathcal{E}_{K}(x, s-x)-z}, \\
K_{23}(z ; x, s)=\frac{(2 \pi)^{-1} \sqrt{\mu_{2} \mu_{3}}}{\mathcal{E}_{K}(s-x, x)-z} .
\end{gathered}
$$

Пусть $n(1, \mathbf{T}(z)), z<\tau(K),-$ число собственных значений (с учетом кратности) оператора $\mathbf{T}(z)$, лежащих правее от 1. Совершенно аналогично доказательству, приведеному в работе [10], пользуясь методами Фредгольма, можно доказать следующую лемму.

ЛЕмма 4. Для числа $N(z)$ (с учетом кратности) собственных значений оператора $H(K)$, лежаших левее от $z<\tau(K)$, имеет место равенство

$$
N(z)=n(1, \mathbf{T}(z)), \quad z<\tau(K)
$$

Перейдем к доказательству теоремы 1. Возможны следующие случаи:

1) $\tau(K)=\lambda_{\alpha}^{\min }(K)<\lambda_{\beta}^{\min }(K) \leqslant \lambda_{\gamma}^{\min }(K),\{\alpha, \beta, \gamma\}=\{1,2,3\}$;

2) $\tau(K)=\lambda_{\alpha}^{\min }(K)=\lambda_{\beta}^{\min }(K)<\lambda_{\gamma}^{\min }(K),\{\alpha, \beta, \gamma\}=\{1,2,3\}$;

3) $\tau(K)=\lambda_{1}^{\min }(K)=\lambda_{2}^{\min }(K)=\lambda_{3}^{\min }(K)$.

Докажем теорему 1 для случая 3 (для случаев 1 и 2 доказательство аналогично). В этом случае существует $x_{\alpha} \in T$ такое, что

$$
\Delta_{\alpha}(x ; \tau(K))=0, \quad \alpha=1,2,3 .
$$

Напомним, что функция $\Delta_{\alpha}(\cdot ; z)$ является аналитической при каждом $z \leqslant \tau(K)$. Поэтому число нулей этой функции конечно.

Обозначим через $p_{1}^{(\alpha)}, \ldots, p_{n_{\alpha}}^{(\alpha)}$ нули функции $\Delta_{\alpha}(\cdot ; \tau(K))$ и $k_{l}^{(\alpha)}-$ кратность нуля $p_{l}^{(\alpha)}, l=1, \ldots, n_{\alpha}$. Пусть $U_{\varepsilon}(a)-\varepsilon$-окрестность точки $a \in T$.

Отметим, что разность $\mathcal{E}_{K}(\cdot ; \cdot)-z$ является положительной при $z \leqslant \tau(K)$, следовательно, функция $K_{\alpha \beta}(z ; \cdot, \cdot)$ аналитична в $T^{2}$. Поэтому существует число $\varepsilon>0$ 
такое, что для всех $\alpha, \beta \in\{1,2,3\}, l \in\left\{1, \ldots, n_{\alpha}\right\}, s \in\left\{1, \ldots, n_{\beta}\right\}$ и $z \leqslant \tau(K)$ имеют место представления

$$
\begin{aligned}
K_{\alpha \beta}(z ; x, y)= & \sum_{k=0}^{\left[k_{l}^{(\beta)} / 2\right]} c_{l k}^{\alpha \beta}(z ; x)\left(y-p_{l}^{(\beta)}\right)^{k}+\left(y-p_{l}^{(\beta)}\right)^{\left[k_{\beta} / 2\right]+1} m_{\alpha \beta}^{(l)}(z ; x, y), \\
x \in T, \quad y \in U_{\varepsilon}\left(p_{l}^{(\beta)}\right), & \\
K_{\alpha \beta}(z ; x, y)= & \sum_{k=0}^{\left[k_{l}^{(\alpha)} / 2\right]} d_{l k}^{\alpha \beta}(z ; y)\left(x-p_{l}^{(\alpha)}\right)^{k}+\left(x-p_{l}^{(\alpha)}\right)^{\left[k_{\alpha} / 2\right]+1} s_{\alpha \beta}^{(l)}(z ; x, y), \\
K_{\alpha \beta}(z ; x, y)= & \sum_{k=0}^{\left[k_{l}^{(\alpha)} / 2\right]\left[k_{s}^{(\beta)} / 2\right]} \sum_{r=0}^{(\beta)} c_{k r}^{\alpha \beta}(l, s, z)\left(x-p_{l}^{(\alpha)}\right)^{k}\left(y-p_{s}^{(\alpha)}\right)^{r}+ \\
& +\sum_{k=0}^{\left[k_{l}^{(\alpha)} / 2\right]} \sum_{r=\left[k_{s}^{(\beta)} / 2\right]+1}^{\infty} c_{k r}^{\alpha \beta}(l, s, z)\left(x-p_{l}^{(\alpha)}\right)^{k}\left(y-p_{s}^{(\alpha)}\right)^{r}+ \\
& +\sum_{k=\left[k_{l}^{(\alpha)} / 2\right]+1}^{\infty} \sum_{r=0}^{\left[k_{s}^{(\beta)} / 2\right]} c_{k r}^{\alpha \beta}(l, s, z)\left(x-p_{l}^{(\alpha)}\right)^{k}\left(y-p_{s}^{(\alpha)}\right)^{r}+ \\
& +\left(x-p_{l}^{(\alpha)}\right)^{\left[k_{\alpha} / 2\right]+1}\left(y-p_{s}^{(\beta)}\right)^{\left[k_{\beta} / 2\right]+1} q_{\alpha \beta}^{(l s)}(z ; x, y), \\
& (x, y) \in U_{\varepsilon}\left(p_{l}^{(\beta)}\right) \times U_{\varepsilon}\left(p_{s}^{(\beta)}\right),
\end{aligned}
$$

где $[a]$ - целая часть числа $a, m_{\alpha \beta}^{(l)}(z ; \cdot, \cdot), s_{\alpha \beta}^{(l)}(z ; \cdot, \cdot)$ и $q_{\alpha \beta}^{(l s)}(z ; \cdot, \cdot)$ - некоторые аналитические функции соответственно на $T \times U_{\varepsilon}\left(p_{l}^{(\beta)}\right), U_{\varepsilon}\left(p_{l}^{(\beta)}\right) \times T$ и $U_{\varepsilon}\left(p_{l}^{(\beta)}\right) \times$ $U_{\varepsilon}\left(p_{s}^{(\beta)}\right)$.

Лемма 5. Существуют $\varepsilon>0$ и $C>0$ такие, что для всех $x \in U_{\varepsilon}\left(p_{l}^{(\alpha)}\right), z \leqslant$ $\tau(K)$ и $l \in\left\{1, \ldots, n_{\alpha}\right\}$ имеет место равенство

$$
\frac{\left|x-p_{l}^{(\alpha)}\right|^{\left[k_{l}^{(\alpha)} / 2\right]+1}}{\sqrt{\Delta_{\alpha}(x ; z)}} \leqslant C .
$$

ДокАЗАТЕЛЬСтво. Функция $\Delta_{\alpha}(x ; \cdot)$ монотонно убывает:

$$
\frac{\left|x-p_{l}^{(\alpha)}\right|^{\left[k_{l}^{(\alpha)} / 2\right]+1}}{\sqrt{\Delta_{\alpha}(x ; z)}} \leqslant \frac{\left|x-p_{l}^{(\alpha)}\right|^{\left[k_{l}^{(\alpha)} / 2\right]+1}}{\sqrt{\Delta_{\alpha}(x ; \tau(K))}} .
$$

Отсюда, заметив, что $k_{l}^{(\alpha)}-$ кратность нуля $p_{l}^{(\alpha)}$ функции $\Delta_{\alpha}(\cdot ; \tau(K))$, и из аналитичности функции $\Delta_{\alpha}(\cdot ; \tau(K))$ получаем неравенство $(5)$.

Лемма 6. Оператор $T_{\alpha \beta}(z), \alpha \neq \beta$, представляется в виде

$$
T_{\alpha \beta}(z)=\widetilde{T}_{\alpha \beta}(z)+F_{\alpha \beta}(z), \quad \alpha \neq \beta,
$$

где операторнозначная функиия $\widetilde{T}_{\alpha \beta}(\cdot)$ непрерьвна по норме на $(-\infty, \tau(K)]$ u $F_{\alpha \beta}(z), z<\tau(K)$, и независимо от $z$ является конечномерным оператором. 
ДоказАтельство. Напомним, что ядро $T_{\alpha \beta}(z ; x, y)$ интегрального оператора $T_{\alpha \beta}(z)$ имеет вид $T_{\alpha \beta}(z ; x, y)=\left[\Delta_{\alpha}(x ; z)\right]^{-1 / 2} K_{\alpha \beta}(z ; x, y)\left[\Delta_{\beta}(y ; z)\right]^{-1 / 2}$. Пользуясь представлением (2)-(4), имеем

$$
T_{\alpha \beta}(z)=\widetilde{T}_{\alpha \beta}(z)+F_{\alpha \beta}(z), \quad \alpha \neq \beta .
$$

Здесь ядра $\widetilde{T}_{\alpha \beta}(z ; x, y)$ и $F_{\alpha \beta}(z ; x, y)$ интегральных операторов $\widetilde{T}_{\alpha \beta}(z)$ и $F_{\alpha \beta}(z)$ соответственно имеют вид

$$
\begin{aligned}
& \widetilde{T}_{\alpha \beta}(z ; x, y)=\left[1-\chi_{\alpha}(x)\right]\left[1-\chi_{\beta}(y)\right] \widetilde{T}_{\alpha \beta}(z ; x, y)+ \\
& +\frac{\left[1-\chi_{\alpha}(x)\right]}{\sqrt{\Delta_{\alpha}(x ; z)}} \sum_{l=0}^{n_{\beta}} \frac{\chi_{\beta}(y)\left(y-p_{l}^{(\beta)}\right)^{\left[k_{l}^{(\beta)} / 2\right]+1}}{\sqrt{\Delta_{\beta}(y ; z)}} M_{\alpha \beta}^{(l)}(z ; x, y)+ \\
& +\frac{\left[1-\chi_{\beta}(y)\right]}{\sqrt{\Delta_{\beta}(y ; z)}} \sum_{l=1}^{n_{\alpha}} \frac{\chi_{\alpha}(x)\left(x-p_{l}^{(\alpha)}\right)^{\left[k_{l}^{(\alpha)} / 2\right]+1}}{\sqrt{\Delta_{\alpha}(x ; z)}} S_{\alpha \beta}^{(l)}(z ; x, y)+ \\
& +\chi_{\alpha}(x) \chi_{\beta}(y) \sum_{l=1}^{n_{\alpha}} \sum_{s=1}^{n_{\beta}} \frac{\left(x-p_{l}^{(\alpha)}\right)^{\left[k_{l}^{(\alpha)} / 2\right]+1}}{\sqrt{\Delta_{\alpha}(x ; z)}} \frac{\left(y-p_{s}^{(\beta)}\right)^{\left[k_{s}^{(\beta)} / 2\right]+1}}{\sqrt{\Delta_{\beta}(y ; z)}} Q_{\alpha \beta}^{(l s)}(z ; x, y) \text {, } \\
& F_{\alpha \beta}(z ; x, y)=\frac{\left[1-\chi_{\alpha}(x)\right]}{\sqrt{\Delta_{\alpha}(x ; z)}} \frac{\chi_{\beta}(y)}{\sqrt{\Delta_{\beta}(y ; z)}} \sum_{s=0}^{n_{\beta}} \sum_{r=0}^{\left[k_{s}^{(\beta)} / 2\right]}\left(y-p_{s}^{(\beta)}\right)^{r} c_{s r}^{\alpha \beta}(z ; x)+ \\
& +\frac{\chi_{\alpha}(x)}{\sqrt{\Delta_{\alpha}(x ; z)}} \frac{\left[1-\chi_{\beta}(y)\right]}{\sqrt{\Delta_{\beta}(y ; z)}} \sum_{l=0}^{n_{\alpha}} \sum_{k=0}^{\left[k_{l}^{(\alpha)} / 2\right]}\left(x-p_{l}^{(\alpha)}\right)^{k} d_{l k}^{\alpha \beta}(z ; y)+ \\
& +\frac{\chi_{\alpha}(x)}{\sqrt{\Delta_{\alpha}(x ; z)}} \frac{\chi_{\beta}(y)}{\sqrt{\Delta_{\beta}(y ; z)}} \sum_{l=0}^{n_{\alpha}} \sum_{s=0}^{n_{\beta}}\left[\sum_{k=0}^{\left[k_{l}^{(\alpha)} / 2\right]} \sum_{r=0}^{\left[k_{s}^{(\beta)} / 2\right]} c_{k r}^{\alpha \beta}(l, s, z)\left(x-p_{l}^{(\alpha)}\right)^{k}\left(y-p_{s}^{(\beta)}\right)^{r}+\right. \\
& +\sum_{k=0}^{\left[k_{l}^{(\alpha)} / 2\right]} \sum_{r=\left[k_{s}^{(\beta)} / 2\right]+1}^{\infty} c_{k r}^{\alpha \beta}(l, s, z)\left(x-p_{l}^{(\alpha)}\right)^{k}\left(y-p_{s}^{(\beta)}\right)^{r}+ \\
& \left.+\sum_{k=\left[k_{l}^{(\alpha)} / 2\right]+1}^{\infty} \sum_{r=0}^{\left[k_{s}^{(\beta)} / 2\right]} c_{k r}^{\alpha \beta}(l, s, z)\left(x-p_{l}^{(\alpha)}\right)^{k}\left(y-p_{s}^{(\beta)}\right)^{r}\right] \text {, }
\end{aligned}
$$

где $\chi_{\alpha}(\cdot)$ - характеристическая функция множества $U_{\alpha}=\bigcup_{l=1}^{n_{\alpha}} U_{\varepsilon}\left(p_{l}^{(\alpha)}\right)$,

$$
\begin{aligned}
M_{\alpha \beta}^{(l)}(z ; x, y) & = \begin{cases}m_{\alpha \beta}^{(l)}(z ; x, y), & (x, y) \in T \times U_{\varepsilon}\left(p_{l}^{(\beta)}\right), \\
0 & \text { в остальных случаях, }\end{cases} \\
S_{\alpha \beta}^{(l)}(z ; x, y) & = \begin{cases}s_{\alpha \beta}^{(l)}(z ; x, y), & (x, y) \in U_{\varepsilon}\left(p_{l}^{(\beta)}\right) \times T, \\
0 & \text { в остальных случаях, }\end{cases} \\
Q_{\alpha \beta}^{(l s)}(z ; x, y) & = \begin{cases}q_{\alpha \beta}^{(l s)}(z ; x, y), & (x, y) \in U_{\varepsilon}\left(p_{l}^{(\beta)}\right) \times U_{\varepsilon}\left(p_{s}^{(\beta)}\right), \\
0 & \text { в остальных случаях. }\end{cases}
\end{aligned}
$$

Из равенства $(5)$ видно, что функция $\widetilde{T}_{\alpha \beta}(z ; \cdot, \cdot)$ является квадратично-интегрируемой на $T^{2}$ при $z \leqslant \tau(K)$ и сходится почти всюду к $\widetilde{T}_{\alpha \beta}(\tau(K) ; \cdot, \cdot)$ при $z \rightarrow \tau(K)$. 
Поэтому по теореме Лебега оператор $\widetilde{T}_{\alpha \beta}(z)$ сходится по норме к $\widetilde{T}_{\alpha \beta}(\tau(K))$ при $z \rightarrow \tau(K)$. Конечномерность оператора $F_{\alpha \beta}(z)$ следует из определения $F_{\alpha \beta}(z ; x, y)$. Лемма 6 доказана.

Определим оператор $\widetilde{\mathbf{T}}(z), z \leqslant \tau(K)$, в гильбертовом пространстве $L_{2}^{(3)}(T)$, заданный матрицей

$$
\widetilde{\mathbf{T}}(z)=\left(\widetilde{T}_{i j}(z)\right)_{i, j=1}^{3} .
$$

Поскольку для каждого ограниченного оператора $\mathbf{A}$, действующего в гильбертовом пространстве $L_{2}^{(3)}(T)$, для любой $\Phi=\left(\varphi_{1}, \varphi_{2}, \varphi_{3}\right) \in L_{2}^{(3)}(T)$ имеют место соотношения

$$
\begin{aligned}
\|\mathbf{A} \Phi\|_{L_{2}^{(3)}(T)}^{2} & =(\mathbf{A} \Phi, \mathbf{A} \Phi)_{L_{2}^{(3)}(T)}=\sum_{i=1}^{3}\left\|(\mathbf{A} \Phi)_{i}\right\|^{2}=\sum_{i=1}^{3}\left\|\sum_{j=1}^{3} A_{i j} \varphi_{j}\right\|^{2} \leqslant \\
& \leqslant \sum_{i=1}^{3} 3 \sum_{j=1}^{3}\left\|A_{i j} \varphi_{j}\right\|^{2} \leqslant 3 \sum_{i=1}^{3} \sup _{j}\left\|A_{i j}(z)\right\|^{2}\|\Phi\|^{2},
\end{aligned}
$$

справедлива оценка

$$
\|\mathbf{A}\| \leqslant \sqrt{3} \sqrt{\sum_{i=1}^{3} \sup _{j}\left\|A_{i j}\right\|^{2}}
$$

Поэтому

$$
\|\widetilde{\mathbf{T}}(z)-\widetilde{\mathbf{T}}(\tau(K))\| \leqslant \sqrt{3} \sqrt{\sum_{j=1}^{3} \sup _{i}\left\|\widetilde{T}_{i j}(z)-\widetilde{T}_{i j}(\tau(K))\right\|^{2}}, \quad z<\tau(K) .
$$

Из неравенства (6) и леммы 6 следует

Лемма 7. Оператор $\mathbf{T}(z)$ представляется в виде

$$
\mathbf{T}(z)=\widetilde{\mathbf{T}}(z)+\mathbf{F}(z)
$$

где операторнозначная функиия $\widetilde{\mathbf{T}}(\cdot)$ непрерывна по норме на $(-\infty, \tau(K)]$ u $\mathbf{F}(z)$, $z<\tau(K)$, независимо от $z$ является конечномерным оператором.

Далее, пользуясь неравенством Вейля [12] $n(a+b, A+B) \leqslant n(a, A)+n(b, B)$ для компактных операторов $A, B$, получим

$$
\begin{aligned}
N(z) \leqslant & n\left(\frac{2}{3}, \widetilde{\mathbf{T}}(z)\right)+n\left(\frac{1}{3}, \mathbf{F}(z)\right) \leqslant n\left(\frac{1}{3}, \widetilde{\mathbf{T}}(z)-\widetilde{\mathbf{T}}(\tau(K))\right)+ \\
& +n\left(\frac{1}{3}, \widetilde{\mathbf{T}}(\tau(K))\right)+n\left(\frac{1}{3}, \mathbf{F}(z)\right) .
\end{aligned}
$$

Согласно лемме 7 оператор $\widetilde{\mathbf{T}}(\tau(K))$ является компактным, поэтому

$$
n\left(\frac{1}{3}, \widetilde{\mathbf{T}}(\tau(K))\right)<\infty
$$


и при малых $\tau(K)-z<0$ имеет место равенство

$$
n\left(\frac{1}{3}, \widetilde{\mathbf{T}}(z)-\widetilde{\mathbf{T}}(\tau(K))\right)=0 .
$$

Поскольку $\mathbf{F}(z)$ есть конечномерный оператор и размерность пространства его значений не зависит от $z, z<\tau(K)$, существует число $F>0$ такое, что для всех $z<\tau(K)$ верно

$$
n\left(\frac{1}{3}, \mathbf{F}(z)\right) \leqslant F<\infty .
$$

Таким образом, для всех $z<\tau(K)$ имеет место неравенство $n(1, \mathbf{T}(z))<\infty$. Следовательно, по лемме 4 число собственных значений $H(K)$, лежащих на $(-\infty, \tau(K))$, является конечным.

Теорема 1 доказана.

\section{4. СПЕКТРЫ ОПЕРАТОРОВ КАНАЛОВ ПРИ $m_{1}=m_{2}=\infty$}

В настоящем разделе мы предполагаем, что $m_{1}=m_{2}=\infty, m_{3}<\infty$. В этом случае функция $\Delta_{\alpha}(x ; z)$ не зависит от $x \in T$. Для удобства обозначим ее через $\Delta_{\alpha}(z)$ :

$$
\Delta_{\alpha}(z)=1-\frac{\mu_{\alpha}}{2 \pi} \int_{-\pi}^{\pi} \frac{d s}{\varepsilon(s) / m_{3}-z}, \quad z \notin[m, M], \quad m=0, \quad M=\frac{2}{m_{3}}, \quad \alpha=1,2 .
$$

Лемма 8. Резольвенты $R_{\alpha}(z)=\left(H_{\alpha}(K)-z I\right)^{-1}$ операторов каналов $H_{\alpha}(K)$, $\alpha=1,2,3$, действуюших в $L_{2}\left(T^{2}\right)$, определяются по формулам

$$
\begin{aligned}
\left(R_{1}(z) f\right)(x, y)= & \frac{f(x, y)}{\varepsilon(K-x-y) / m_{3}-z}+\frac{\left[2 \pi \Delta_{1}(z)\right]^{-1} \mu_{3}}{\varepsilon(K-x-y) / m_{3}-z} \times \\
& \times \int_{-\pi}^{\pi} \frac{f(s, y) d s}{\varepsilon(K-s-y) / m_{3}-z}, \quad z \notin[m, M], \quad \Delta_{1}(z) \neq 0 ; \\
\left(R_{2}(z) f\right)(x, y)= & \frac{f(x, y)}{\varepsilon(K-x-y) / m_{3}-z}+\frac{\left[2 \pi \Delta_{2}(z)\right]^{-1} \mu_{3}}{\varepsilon(K-x-y) / m_{3}-z} \times \\
& \times \int_{-\pi}^{\pi} \frac{f(x, s) d s}{\varepsilon(K-x-s) / m_{3}-z}, \quad z \notin[m, M], \quad \Delta_{2}(z) \neq 0 ; \\
\left(R_{3}(z) f\right)(x, y)= & \frac{f(x, y)}{\varepsilon(K-x-y) / m_{3}-z}+ \\
& +\frac{(2 \pi)^{-1}}{\varepsilon(K-x-y) / m_{3}-z} \frac{1}{\varepsilon(K-x-y) / m_{3}-\mu_{3}-z} \times \\
& \times \int_{-\pi}^{\pi} f(s, x+y-s) d s, \quad z \notin[m, M] \cup \Lambda_{3} .
\end{aligned}
$$

ДокАЗАТЕЛьство. Докажем лемму для случая $\alpha=3$. Рассмотрим резольвентное уравнение

$$
\left(H_{3}(K)-z I\right) f=g
$$


для заданного $g \in L_{2}\left(T^{2}\right)$. Отсюда, заметив, что $\varepsilon(x) / m_{3}-z \neq 0$ при $z \notin[m, M] \cup \Lambda_{3}$ и $x \in T$, получим

$$
f(x, y)=\frac{c(x+y)}{\varepsilon(K-x-y) / m_{3}-z}+\frac{g(x, y)}{\varepsilon(K-x-y) / m_{3}-z},
$$

где

$$
c(x+y)=\frac{\mu_{3}}{2 \pi} \int_{-\pi}^{\pi} f(s, x+y-s) d s .
$$

Подставляя это выражение в уравнение (7), получаем

$$
c(x+y)=c(x+y) \frac{\mu_{3}}{2 \pi} \int_{-\pi}^{\pi} \frac{d s}{\varepsilon(K-p-q) / m_{3}-z}+\frac{\mu_{3}}{2 \pi} \int_{-\pi}^{\pi} \frac{g(s, x+y-s)}{\varepsilon(K-x-y) / m_{3}-z} .
$$

Отсюда, учитывая $\varepsilon(x) / m_{3}-\mu_{3}-z \neq 0$ при $z \notin[m, M] \cup \Lambda_{3}$ и $x \in T$, находим

$$
c(x+y)=\frac{1}{\varepsilon(K-x-y) / m_{3}-\mu_{3}-z} \frac{\mu_{3}}{2 \pi} \int_{-\pi}^{\pi} g(s, x+y-s) d s .
$$

Подставляя последнее равенство в уравнение $(7)$, имеем $f(x, y)=\left(R_{3}(z) g\right)(x, y)$.

Случаи $\alpha=1,2$ доказываются аналогично.

ДокаЗАтеЛьство леммы 3. Пусть $z \notin[m, M] \cup \Lambda_{3}\left(z \notin[m, M], \Delta_{i}(z) \neq 0\right.$, $i=1,2)$. Тогда из леммы 8 следует, что $z$ является регулярной точкой оператора $H_{3}(K)\left(H_{i}(K), i=1,2\right)$. Следовательно,

$$
\sigma\left(H_{3}(K)\right) \subset[m, M] \cup \Lambda_{3}, \quad \sigma\left(H_{i}(K)\right) \subset[m, M] \cup\left\{z: \Delta_{i}(z)=0\right\}, \quad i=1,2 .
$$

Заметим, что имеют место включения [11]

$$
[m, M] \subset \sigma\left(H_{\alpha}(K)\right), \quad \alpha=1,2,3 .
$$

Рассмотрим уравнения для собственных функций

$$
H_{i}(K) f_{i}=z f_{i}, \quad z \notin[m, M], \quad i=1,2 .
$$

Отсюда

$$
f_{1}(x, y)=\frac{c_{1}(y)}{\varepsilon(K-x-y) / m_{3}-z}, \quad f_{2}(x, y)=\frac{c_{2}(x)}{\varepsilon(K-x-y) / m_{3}-z},
$$

где

$$
c_{1}(y)=\frac{\mu_{1}}{2 \pi} \int_{-\pi}^{\pi} f(s, y) d s, \quad c_{2}(x)=\frac{\mu_{2}}{2 \pi} \int_{-\pi}^{\pi} f(x, s) d s .
$$

Подставляя выражения (9) в формулы (10), имеем

$$
c_{i}(y) \Delta_{i}(z)=0, \quad i=1,2 .
$$

Отсюда следует, что если число $z \notin[m, M]$ является собственным значением $H_{i}(K)$, $i=1,2$, то $c_{i}(\cdot) \neq 0$ и, значит, $\Delta_{i}(z)=0$. Обратно, если $\Delta_{i}(z)=0$, то функция

$$
f_{1}(x, y)=\frac{\alpha_{1}(y)}{\varepsilon(K-x-y) / m_{3}-z} \quad\left(f_{2}(x, y)=\frac{\alpha_{2}(x)}{\varepsilon(K-x-y) / m_{3}-z}\right)
$$


является собственной функцией $H_{1}(K)\left(H_{2}(K)\right)$, где $\alpha_{i}(\cdot), i=1,2,-$ любая непрерывная функция, определенная в $T$.

Функция $\Delta_{i}(\cdot)$ является монотонной в $(-\infty, m)$ и

$$
\lim _{z \rightarrow-\infty} \Delta_{i}(z)=1, \quad \lim _{z \rightarrow m} \Delta_{i}(z)=-\infty .
$$

Следовательно, функция $\Delta_{i}(\cdot)$ имеет единственный нуль $z=\lambda_{i}, i=1,2$.

Для оператора $H_{3}(K)$ подпространство $\mathcal{H}_{0}=\left\{f \in L_{2}\left(T^{2}\right): f(p, q)=g(p+q)\right\}$ пространства $L_{2}\left(T^{2}\right)$ является инвариантным для $H_{3}(K)$, и оператор $H_{3}(K)$ действует как оператор умножения:

$$
\left(H_{3}(K) g\right)(p+q)=\left[\frac{1}{m_{3}} \varepsilon(K-p-q)-\mu_{3}\right] g(p+q), \quad g \in \mathcal{H} .
$$

Отсюда следует, что $\Lambda_{3} \subset \sigma\left(H_{3}(K)\right)$.

\section{5. ДИСКРЕТНЫЙ СПЕКТР ОПЕРАТОРА $H(K)$ ПРИ $m_{1}=m_{2}=\infty$}

В этом разделе мы предполагаем, что $m_{1}=m_{2}=\infty$. Тогда оператор $H(K)$ оставляет каждое из подпространств $\mathcal{H}_{0}=\left\{f \in L_{2}\left(T^{2}\right): f(p, q)=g(p+q)\right\}$ и $\mathcal{H}_{0}^{\perp}=$ $L_{2}\left(T^{2}\right) \ominus \mathcal{H}_{0}$ инвариантным. При этом

$$
\sigma_{\text {disc }}(H(K))=\sigma_{\text {disc }}\left(H^{0}\right) \cup \sigma_{\text {disc }}\left(H^{1}\right),
$$

где $H^{0}=P H(K) P, H^{1}=P^{\perp} H(K) P^{\perp}, P$ - ортогональный проектор в $\mathcal{H}_{0}, P^{\perp}=$ $I-P$.

Поскольку $\mathcal{H}_{0}$ изоморфно $L_{2}(T)$, оператор $H^{0}$ унитарно-эквивалентен оператору $\bar{H}^{0}$, действующему в $L_{2}(T)$ по формуле

$$
\bar{H}^{0}=E_{0}(K)-\mu_{3} I-\left(\mu_{1}+\mu_{2}\right) v,
$$

где $E_{0}(K)$ - оператор умножения на функцию $\varepsilon(K-\cdot) / m_{3}, I$ - единичный оператор, $v$ - оператор, действующий по формуле (1).

Оператор $H^{1}$ действует в $\mathcal{H}_{0}^{\perp}$ по формуле

$$
H^{1}=H_{0}(K)-\mu_{1} V_{1}-\mu_{2} V_{2} .
$$

Лемма 9. Число $z \notin \sigma_{\mathrm{ess}}\left(H^{1}\right)$ является собственным значением оператора $H^{1}$ тогда и только тогда, когда $D(z)=0$, где

$$
\begin{gathered}
D(z)=\prod_{k=0}^{\infty} D_{k}(z), \\
D_{k}(z)=1-\frac{1}{4 \pi^{2}} \frac{\mu_{1}}{\Delta_{2}(z)} \frac{\mu_{2}}{\Delta_{1}(z)} d_{k}^{2}(z), \quad d_{k}(z)=\int_{-\pi}^{\pi} \frac{e^{-i k s} d s}{\varepsilon(s) / m_{3}-z}, \quad k \in Z .
\end{gathered}
$$

Если число $z_{k} \notin \sigma_{\mathrm{ess}}\left(H^{1}\right)$ - собственное значение $H^{1}$ с $D_{k}\left(z_{k}\right)=0$, то соответствующая собственная функция имеет вид

$f(x, y)=\frac{1}{\varepsilon(K-x-y) / m_{3}-z}\left[\mu_{1} e^{ \pm i k(K / 2-y)} d_{k}\left(z_{k}\right)+\mu_{2} \frac{\mu_{1}}{2 \pi \Delta_{2}(z)} d_{k}\left(z_{k}\right) e^{ \pm i k(K / 2-x)}\right]$,

где $k$ - некоторое натуральное число. 
ДокАЗАтельство. Пусть $z \notin \sigma_{\text {ess }}\left(H^{1}\right)$ - собственное значение оператора $H^{1}$ и $f-$ соответствующий собственный вектор, т. е. уравнение $H_{12}(K) f=z f$ имеет нетривиальное решение $f$. Так как при $z \notin \sigma_{\text {ess }}\left(H^{1}\right)$ оператор $R_{0}(z)$ ограничен в $L_{2}\left(T^{2}\right)$, имеем

$$
f=R_{0}(z)\left[\mu_{1} V_{1}+\mu_{2} V_{2}\right] f .
$$

Отсюда следует, что число $z$ является собственным значением оператора $H^{1}$ тогда и только тогда, когда 1 является собственным значением $R_{0}(z)\left[\mu_{1} V_{1}+\mu_{2} V_{2}\right]$, причем кратности z и 1 совпадают. Введем обозначения

$$
\widetilde{\varphi}_{\alpha}=V_{\alpha} f, \quad \alpha=1,2 .
$$

Из равенства (12) имеем

$$
f(x, y)=\frac{1}{\varepsilon(K-p-q) / m_{3}-z}\left[\mu_{1} \widetilde{\varphi}_{1}(y)+\mu_{2} \widetilde{\varphi}_{2}(x)\right] .
$$

Подставляя выражение (14) в (13), получим, что функции $\widetilde{\varphi}_{\alpha}, \alpha=1,2$, удовлетворяют системе интегральных уравнений

$$
\begin{aligned}
& \widetilde{\varphi}_{1}(y)=\frac{\mu_{1}}{2 \pi} \int_{-\pi}^{\pi} \frac{\widetilde{\varphi}_{1}(y) d s}{\varepsilon(K-y-s) / m_{3}-z}+\frac{\mu_{2}}{2 \pi} \int_{-\pi}^{\pi} \frac{\widetilde{\varphi}_{2}(s) d s}{\varepsilon(K-y-s) / m_{3}-z}, \\
& \widetilde{\varphi}_{2}(x)=\frac{\mu_{1}}{2 \pi} \int_{-\pi}^{\pi} \frac{\widetilde{\varphi}_{1}(s) d s}{\varepsilon(K-s-x) / m_{3}-z}+\frac{\mu_{2}}{2 \pi} \int_{-\pi}^{\pi} \frac{\widetilde{\varphi}_{2}(x) d s}{\varepsilon(K-s-x) / m_{3}-z} .
\end{aligned}
$$

Пользуясь периодичностью подынтегральных функций в этой системе уравнений, производя замену переменных $K / 2-s=t$ в первом и втором интегралах правой части равенств и заметив, что $\Delta_{\alpha}(z) \neq 0, \alpha=1,2$, при $z \notin \sigma_{\mathrm{ess}}\left(H_{12}(K)\right)$, убедимся, что $\varphi_{\alpha}, \alpha=1,2$, удовлетворяют следующей системе интегральных уравнений:

$$
\begin{aligned}
& \varphi_{1}(y)=\frac{1}{\Delta_{1}(z)} \frac{\mu_{2}}{2 \pi} \int_{-\pi}^{\pi} \frac{\varphi_{2}(t) d t}{\varepsilon(y+t) / m_{3}-z}, \\
& \varphi_{2}(x)=\frac{1}{\Delta_{2}(z)} \frac{\mu_{1}}{2 \pi} \int_{-\pi}^{\pi} \frac{\varphi_{1}(t) d t}{\varepsilon(t+x) / m_{3}-z}, \quad \varphi_{i}(y)=\widetilde{\varphi}_{i}\left(y+\frac{K}{2}\right) .
\end{aligned}
$$

При этом вектор $f$, определенный по формуле (14), является собственным вектором оператора $H_{12}(K)$, соответствующим собственному числу $z \notin \sigma_{\mathrm{ess}}\left(H_{12}(K)\right)$, тогда и только тогда, когда $\Phi=\left(\varphi_{1}, \varphi_{2}\right)$ является решением системы (15). Число таких линейно независимых собственных векторов $f$ и $\Phi$ совпадает.

Подставляя второе из равенств (15) в первое, получаем

$$
\begin{gathered}
\varphi_{1}(y)=\frac{1}{\Delta_{1}(z) \Delta_{2}(z)} \frac{\mu_{1} \mu_{2}}{4 \pi^{2}} \int_{-\pi}^{\pi} \int_{-\pi}^{\pi} \frac{1}{\varepsilon(y+t) / m_{3}-z} \frac{\varphi_{1}(s) d t d s}{\varepsilon(s+t) / m_{3}-z}, \\
\varphi_{i}(y)=\widetilde{\varphi}_{i}\left(y+\frac{K}{2}\right),
\end{gathered}
$$

или

$$
\varphi_{1}=Q(z) \varphi_{1}, \quad Q(z)=\frac{1}{\Delta_{1}(z) \Delta_{2}(z)} \frac{\mu_{1} \mu_{2}}{4 \pi^{2}} Q Q^{*}
$$


$Q$ - интегральный оператор, действующий в $L_{2}(T)$ по формуле

$$
(Q \varphi)(x)=\int_{-\pi}^{\pi} \frac{\varphi(t)}{\varepsilon(x+t) / m_{3}-z} .
$$

При этом если $\Phi=\left(\varphi_{1}, \varphi_{2}\right)$ является решением системы $(15)$, то $\varphi_{1}$ является собственной функцией оператора $Q(z)$, соответствующей собственному значению 1. Обратно, если $\varphi_{1}$ является собственной функцией оператора $Q(z)$, соответствующей собственному значению 1 , то $\Phi=\left(\varphi_{1}, \varphi_{2}\right), \varphi_{2}=\left(1 / \Delta_{2}(z)\right)\left(\mu_{1} / 2 \pi\right) Q \varphi_{1}$ является решением системы (15). Число таких линейно независимых собственных векторов $\varphi_{1}$ и $\Phi$ совпадает.

Так как функция $1 /\left(\varepsilon(\cdot) / m_{3}-z\right)$ непрерывна на $T$, то оператор $Q$ является оператором Гильберта-Шмидта. Следовательно, оператор $Q(z)$ принадлежит классу операторов со следом.

Поэтому детерминант $\operatorname{det}(I-Q(z))$ оператора $I-Q(z)$ существует и определяется по формуле (см. теорему XIII.106 в книге [13])

$$
\operatorname{det}(I-Q(z))=\prod_{k=0}^{\infty}\left(1-\lambda_{k}(z)\right)
$$

где $\left\{\lambda_{k}(z)\right\}$ - собственные значения $Q(z)$, выписанные с учетом алгебраической кратности. По теореме XIII.105 книги [13] число 1 есть собственное значение оператора $Q(z)$ тогда и только тогда, когда $\operatorname{det}(I-Q(z))=0$.

Пусть $\varphi$ - собственная функция оператора $Q(z)$, соответствующая собственному значению $\lambda$, т. е.

$$
\lambda \varphi(y)=\frac{1}{\Delta_{1}(z) \Delta_{2}(z)} \frac{\mu_{1} \mu_{2}}{4 \pi^{2}} \int_{-\pi}^{\pi} \int_{-\pi}^{\pi} \frac{1}{\varepsilon(y+t) / m_{3}-z} \frac{\varphi(s) d t d s}{\varepsilon(s+t) / m_{3}-z} .
$$

Разлагая функцию $\varphi$ в ряд по базису $\left\{e^{i k x}\right\}_{k \in Z}$ и учитывая периодичность подынтегральной функции, получим

$$
c_{k} \lambda e^{i k x}=c_{k} e^{i k x} \frac{1}{\Delta_{1}(z) \Delta_{2}(z)} \frac{\mu_{1} \mu_{2}}{4 \pi^{2}}\left|\int_{-\pi}^{\pi} \frac{1}{\varepsilon(s) e^{i k s} d s / m_{3}-z}\right|^{2},
$$

или

$$
\lambda(z)=\frac{1}{\Delta_{1}(z) \Delta_{2}(z)} \frac{\mu_{1} \mu_{2}}{4 \pi^{2}}\left|d_{k}(z)\right|^{2} .
$$

Таким образом, собственное значение $\lambda_{k}(z), k \in Z$, оператора $Q(z)$, участвующее в формуле (17), имеет вид

$$
\lambda_{k}(z)=\frac{1}{\Delta_{1}(z) \Delta_{2}(z)} \frac{\mu_{1} \mu_{2}}{4 \pi^{2}}\left|d_{k}(z)\right|^{2},
$$

а $\psi_{k}(x)=e^{i k x}, k \in Z$, является собственной функцией оператора $Q(z)$, соответствующей собственному значению $\lambda_{k}(z), k \in Z$.

Заметим, что $d_{k}(z)=d_{-k}(z)$ для любого натурального $k$ и $z \notin \sigma_{\mathrm{ess}}\left(H_{12}(K)\right)$. Поэтому, если число $1=\lambda_{k}\left(z_{k}\right), z_{k} \notin \sigma_{\mathrm{ess}}\left(H_{12}(K)\right)$, - собственное значение $Q\left(z_{k}\right)$, то $\varphi_{k}=e^{ \pm i k x}$ является соответствующей собственной функцией оператора $Q\left(z_{k}\right)$. 
Отсюда если число $z_{k} \notin \sigma_{\text {ess }}\left(H_{12}(K)\right)$ - собственное значение $H_{12}(K)$ с $D_{k}\left(z_{k}\right)=$ $1-\lambda_{k}\left(z_{k}\right)=0$, то из формул (14) и (15) получаем соответствующую собственную функцию

$f(x, y)=\frac{1}{\varepsilon(K-x-y) / m_{3}-z}\left[\mu_{1} e^{ \pm i k(K / 2-y)} d_{k}\left(z_{k}\right)+\mu_{2} \frac{\mu_{1}}{2 \pi \Delta_{2}(z)} d_{k}\left(z_{k}\right) e^{ \pm i k(K / 2-x)}\right]$.

Лемма 9 доказана.

Лемма 10. 1. Имеют место равенства

$$
\begin{aligned}
\Delta_{\alpha}(z) & =1-\frac{\mu_{\alpha} \sqrt{m_{3}}}{\sqrt{2-m_{3} z}} \frac{1}{\sqrt{-z}}, \quad \alpha=1,2, \\
d_{k}(z) & =\frac{2 \pi m_{3}\left[1-m_{3} z-\sqrt{\left(m_{3} z\right)^{2}-2 m_{3} z}\right]^{k}}{\sqrt{\left(m_{3} z\right)^{2}-2 m_{3} z}} .
\end{aligned}
$$

2. Обозначим через $z=\lambda_{i}$ отрицательный ноль функции $\Delta_{i}(\cdot), i=1,2$, и положим $\lambda_{\min }=\min \left\{\lambda_{1}, \lambda_{2}\right\}, \lambda_{\max }=\max \left\{\lambda_{1}, \lambda_{2}\right\}$. Тогда функция $D_{k}(\cdot)$ имеет ноль $z_{k} \in\left(-\infty, \lambda_{\min }\right)$ при каждом $k=0,1, \ldots$, a также существует последовательность натуральных чисел $\left\{k_{n}\right\}$ и число $\xi_{k_{n}} \in\left(\lambda_{\max }, 0\right)$ maкие, что $D_{k_{n}}\left(\xi_{k_{n}}\right)=0$ при каждом $n=0,1, \ldots$. При этом

$$
\lim _{k \rightarrow \infty} z_{k}=\lambda_{\min }, \quad \lim _{n \rightarrow \infty} \xi_{k_{n}}=\lambda_{\max }
$$

ДокАзАтЕЛЬСтво. 1. Используя известное равенство

$$
\int_{0}^{\pi} \frac{\cos (k t) d t}{1-2 a \cos t+a^{2}}=\frac{\pi a^{k}}{1-a^{2}}
$$

где $k=0,1, \ldots$ и $0<a<1$, имеем

$$
\int_{0}^{\pi} \frac{\cos (k t) d t}{1-\left(2 a /\left(1+a^{2}\right)\right) \cos t}=\frac{\pi\left(1+a^{2}\right) a^{k}}{1-a^{2}} .
$$

С учетом равенства

$$
\frac{\left(1-a^{2}\right)^{2}}{\left(1+a^{2}\right)^{2}}=1-\frac{(2 a)^{2}}{\left(1+a^{2}\right)^{2}}
$$

формула (19) имеет вид

$$
\int_{0}^{\pi} \frac{\cos (k t) d t}{1-\left(2 a /\left(1+a^{2}\right)\right) \cos t}=\frac{\pi a^{k}}{\sqrt{1-(2 a)^{2} /\left(1+a^{2}\right)^{2}}} .
$$

Так как $\varepsilon(\cdot)$ - четная функция, то $d_{k}(z)$ принимает вид

$$
d_{k}(z)=2 \int_{0}^{\pi} \frac{\cos (k s) d s}{(1-\cos s) / m_{3}-z}=\frac{2 m_{3}}{1-m_{3} z} \int_{0}^{\pi} \frac{\cos (k s) d s}{1-\cos s /\left(1-m_{3} z\right)}
$$

Введя обозначение $b=1-m_{3} z-\sqrt{\left(m_{3} z\right)^{2}-2 m_{3} z}$, получаем

$$
d_{k}(z)=\frac{2 m_{3}}{1-m_{3} z} \int_{0}^{\pi} \frac{\cos (k s) d s}{1-2 b \cos s /\left(1+b^{2}\right)} .
$$


Отсюда с учетом включения $b \in(0,1)$ и выражения $(20)$ следует

$$
d_{k}(z)=\frac{2 m_{3} \pi\left[1-m_{3} z-\sqrt{\left(m_{3} z\right)^{2}-2 m_{3} z}\right]^{k}}{\sqrt{\left(m_{3} z\right)^{2}-2 m_{3} z}}, \quad k=0,1,2, \ldots
$$

Поскольку $\Delta_{\alpha}(z)=1-\left(\mu_{\alpha} / 2 \pi\right) d_{0}(z)$, получаем

$$
\Delta_{\alpha}(z)=1-\frac{\mu_{\alpha} \sqrt{m_{3}}}{\sqrt{2-m_{3} z}} \frac{1}{\sqrt{-z}}, \quad \alpha=1,2 .
$$

2. Для каждого $k=0,1, \ldots$ имеют место равенства

$$
\lim _{z \rightarrow-\infty} D_{k}(z)=1, \quad \lim _{z \rightarrow \lambda_{\min }-0} D_{k}(z)=-\infty .
$$

Отсюда согласно непрерывности $D_{k}(\cdot)$ следует существование $z_{k} \in\left(-\infty, \lambda_{\min }\right)$ такого, что $D_{k}\left(z_{k}\right)=0$. Поскольку $\lim _{z \rightarrow-\infty} D(z)=1$ и $D(\cdot)$ - аналитическая функция на $\mathbf{C} \backslash\left[\left\{\lambda_{1}\right\} \cup\left\{\lambda_{2}\right\} \cup\left[0,2 / m_{3}\right]\right]$ (где $\mathbf{C}-$ комплексная плоскость), то должно быть $\lim _{k \rightarrow \infty} z_{k}=\lambda_{\min }$.

Отметим, что функция $D_{k}(\cdot)$ определена на $\left(\lambda_{\max }, 0\right)$ и

$$
0<1-m_{3} z-\sqrt{\left(m_{3} z\right)^{2}-2 m_{3} z}<1
$$

для всех $z<0$. Поэтому для каждого фиксированного $z \in\left(\lambda_{\max }, 0\right)$

$$
\lim _{k \rightarrow \infty} D_{k}(z)=1 \text {. }
$$

Следовательно, существует подпоследовательнось $\left\{k_{n}\right\}$ натуральных чисел такая, что $D_{k_{n}}\left(\lambda_{\max } / 2\right)>0$. Далее, заметив, что $\lim _{z \rightarrow \lambda_{\max }-0} D_{k}(z)=-\infty$, из непрерывности функции $D_{k}(\cdot)$ получаем $D_{k_{n}}\left(\xi_{k_{n}}\right)=0$ для некоторого $\xi_{k_{n}} \in\left(\lambda_{\max }, \lambda_{\max } / 2\right)$.

Из аналитичности функции $D(\cdot)$ на $\mathbf{C} \backslash\left[\left\{\lambda_{1}\right\} \cup\left\{\lambda_{2}\right\} \cup\left[0,2 / m_{3}\right]\right]$ следует, что $\lim _{k \rightarrow \infty} \xi_{n_{k}}=\lambda_{\max }$. Лемма 10 доказана.

Аналогично лемме 9 доказывается

Лемма 11. Для всякого $\mu_{i}>0, i=1,2,3$, оператор $\bar{H}^{0}$ имеет единственное собственное значение $\lambda_{3}<\inf \sigma_{\mathrm{ess}}\left(\bar{H}^{0}\right)$ с $\Delta_{3}\left(\lambda_{3}\right)=0$, где

$$
\Delta_{3}(z)=1-\frac{\mu_{1}+\mu_{2}}{2 \pi} \int_{-\pi}^{\pi} \frac{d s}{\varepsilon(s) / m_{3}-\mu_{3}-z}, \quad k \in Z .
$$

Доказательство теоремы 2 следует из формулы (11) и лемм 9-11.

Благодарности. Авторы выражают благодарность рецензенту за полезные замечания.

\section{Список литературы}

[1] В. Н. Ефимов, ЯФ, 12:5 (1970), 1080-1091.

[2] R. D. Amado, J. V. Noble, Phys. Rev. D, 5:8 (1972), 1992-2002.

[3] С. П. Меркурьев, Л. Д. Фаддеев, Квантовая теория рассеяния для систем нескольких частии, Наука, М., 1985. 
[4] Д. Р. Яфаев, Матем. сб., 94(136):4(8) (1974), 567-593.

[5] Yu. N. Ovchinnikov, I. M. Sigal, Ann. Phys., 123:2 (1979), 274-295.

[6] H. Tamura, J. Funct. Anal., 95:2 (1991), 433-459.

[7] С. Н. Лакаев, ТМФ, 89:1 (1991), 94-104.

[8] С. Н. Лакаев, М.Э. Муминов, ТМФ, 135:3 (2003), 478-503.

[9] С. Н. Лакаев, Ж. И. Абдуллаев, Функи. анализ и его прил., 33:2 (1999), 84-88.

[10] М.Э. Муминов, ТМФ, 159:2 (2009), 299-317.

[11] S. Albeverio, S. N. Lakaev, Z. I. Muminov, Ann. Henri Poincaré, 5:4 (2004), 743-772.

[12] М.Ш. Бирман, М.З. Соломяк, Спектралъная теория самосопряженных операторов в гилъбертовом пространстве, Изд-во ЛГУ, Л., 1980.

[13] М. Рид, Б. Саймон, Методы современной математической физики, т. 4: Анализ операторов, Мир, М., 1982.

Поступила в редакцию 24.05.2011, после доработки 6.09.2011 